\title{
Aplicación de técnicas de desenvolvimiento de producto para el desarrollo de un robot antropomórfico
}

\section{Application of product development techniques for the development of an anthropomorphic robot}

\author{
Cristhian Riaño-Jaimes ${ }^{1}$, César Peña-Cortés ${ }^{2}$, Heller Sánchez-Acevedo ${ }^{3}$ \\ ${ }^{1}$ Departamento de Ingeniería Mecánica y Mecatrónica, Universidad de Brasilia, Brasil. Email: cristhianivanrj@aluno.unb.br \\ ${ }^{2}$ Programa de Ingeniería Mecatrónica, Universidad de Pamplona, Colombia. Email: cesarapc@ unipamplona.edu.co \\ ${ }^{3}$ Grupo de Investigación en Energía y Medio Ambiente-GIEMA, Escuela de Ingeniería Mecánica, Universidad Industrial de \\ Santander, Colombia. Email: hgsanche@uis.edu.co
}

ReCiBIDO: Marzo 27, 2017. ACEPTADO: Agosto 30, 2017. Versión FinAL: Septiembre 10, 2017.

\section{RESUMEN}

Los proyectos robóticos involucran componentes enmarcadas dentro de áreas como mecánica, electrónica control y programación en las que intervienen diferentes variables críticas que pueden comprometer la obtención de los resultados esperados. En este proyecto un gran número de requerimientos fueron formulados para ser satisfechos con recursos limitados, lo que obliga a tener un escaso margen de error en su fabricación. Técnicas usadas en el desenvolvimiento de producto fueron empleadas para conseguir cumplir con las especificaciones exigidas para el proyecto. El artículo describe el desarrollo de un robot en las etapas de proyecto informacional, proyecto conceptual, proyecto detallado y fabricación. Se presentan las consideraciones de diseño estudiadas y analizadas para obtener un brazo robótico funcional que ejecuta tareas de agricultura. Fueron empleadas técnicas como QFD (Quality Function Development) para trasladar las necesidades asociadas al proyecto en decisiones de ingeniería. Se emplea el diagrama de Mudge (matriz de análisis de valor) como método de comparación y jerarquización de los requisitos. Con la aplicación de QFD los requerimientos del robot son transformados en características de calidad que permiten generar un proyecto conceptual con los métodos y actividades a ejecutar. Los resultados obtenidos mostraron la efectividad de emplear herramientas de diseño para concebir proyectos robóticos y focalizar los recursos en satisfacer los requerimientos.

PALABRAS CLAVE: Arquitectura sustentable; diseño conceptual; QFD (despliegue de la función de calidad); robótica; TRIZ (teoría de la resolución de problemas inventiva).

\footnotetext{
ABSTRACT

Robotic projects involve components framed within areas such as electronics; mechanics, control and programming in which different critical variables intervene that can compromise the achievement of the expected results. In this project, numerous requirements were formulated to be satisfied with limited resources, which forces to have a small margin of error in its manufacture. Techniques used in product development were used to achieve the required specifications for the project. The article describes the development of a robot in the stages of informational project, conceptual project, detailed project and manufacturing. They are presented design considerations studied and analyzed to obtain a

ISSN Impreso: 1657 - 4583, En Línea: 2145 - 8456

Este artículo puede compartirse bajo la licencia CC BY-ND 4.0 y se referencia usando el siguiente formato: C. Riaño-Jaimes, C. Peña-Cortés, H. G. Sánchez-Acevedo, "Aplicación de técnicas de desenvolvimiento de producto para el desarrollo de un robot antropomórfico," Rev. UIS Ing., Vol. 17, no. 1, pp. 21-34, 2018. Doi: https://doi.org/10.18273/revuin.v17n1-2018002
} 
functional robotic arm running tasks agriculture. Techniques such as QFD (Quality Function Development) were used to translate the needs associated with the project into engineering decisions. The Mudge diagram (value analysis matrix) is used as a method of comparison and hierarchy of requirements. With the application of QFD the requirements of the robot are transformed into quality characteristics that allow to generate a conceptual project with the methods and activities to execute. The results showed the effectiveness of using design tools to create robotic systems and to focus resources on satisfying the requirements.

KEYWORDS: Conceptual design; QFD (Quality Function Development); sustainable architecture; robotics; TRIZ (theory of inventive problem solving).

\section{INTRODUCCIÓN}

La mayoría de los problemas que enfrenta el ser humano están siendo abordados desde distintas perspectivas tecnológicas. El campo de la robótica aporta soluciones para distintos sectores que van desde la parte de robótica de servicios: aplicaciones médicas [1], plataformas móviles [2-3], educacional, espacial, entre muchos otras hasta el sector industrial, que recibe grandes recursos destinados para el desarrollo de proyectos. El problema común que enfrenta el desarrollo de un nuevo robot consiste principalmente en encontrar un conjunto de acciones para ejecutar, que proporcionen una solución que satisfaga las necesidades del problema, buscando el mejor aprovechamiento de los recursos disponibles.

Los esfuerzos iniciales en este proyecto se centraron en generar un plan de acciones que permitan desarrollar un robot que ejecute tareas agrícolas [4]. El problema de diseño se torna complejo cuando en un sistema robótico existen soluciones fuertemente acopladas como son por ejemplo la relación entre peso de un eslabón (componente mecánica) y el torque que debe garantizar el lazo de control de posición (componente electrónica y control). El grado de correlación entre ellas hace necesario emplear metodologías que faciliten la fragmentación del problema y encontrar los principios de solución que permitan satisfacer los dos requerimientos. El sistema robótico propuesto está compuesto por tres grandes componentes, mecánica, electrónica y control; abordadas de manera global y llevadas a solución de detalle.

El concepto de desenvolvimiento de producto ya tiene un recorrido de expansión a lo largo de sus últimos 35 años por la efectividad demostrada en la concepción de productos de consumo [5]. Resultados han sido ampliamente publicados y trasladados a otros escenarios de aplicación [6]. El objetivo perseguido es obtener por medio de herramientas y metodologías de diseño de producto, un planeamiento para el desarrollo del robot. El planeamiento conducirá la ejecución de las etapas de diseño, desarrollo y fabricación usando los recursos disponibles, focalizando los esfuerzos, reduciendo el tiempo en encontrar soluciones, evitando pérdidas, y especialmente cumpliendo con los objetivos trazados para la investigación.

La fase de estudio y diseño en el ciclo de vida de un producto es quizás la más importante y la que requiere mayor atención. Los resultados de esta fase definirán las actividades a ejecutar y el éxito del proyecto. Es importante hacer una investigación exhaustiva y constante para encontrar distintas alternativas de solución. Esta investigación parte de una revisión sobre soluciones robóticas integradas en la agricultura, considerando distintas configuraciones robóticas como son: móviles, de cadena cinemática abierta y cadena cinemática cerrada de las que se puedan extraer experiencias.

La siguiente fase del proyecto "sistema robótico para aplicaciones agrícolas" es concebir un robot con múltiples tareas que se integre perfectamente con otras tecnologías y sirva de plataforma para generar soluciones robóticas en el área agrícola.

La "arquitectura sustentable" o también llamada "arquitectura verde" que consiste principalmente en integrar recursos naturales con edificaciones de manera sostenible, demanda para su masificación grandes lotes de cultivos con diversos tipos de plantas. Se perseguí en este proyecto concebir una plataforma robótica aplicada para asistir de forma autónoma un cultivo de plantas con posibilidad de escalamiento.

En este documento se presenta de manera detallada las actividades de estudio y diseño desarrolladas para crear el robot agrícola, usando metodologías de diseño de producto QFD y métodos para búsqueda de soluciones como el TRIZ (theory of inventive problem solving). En la sección dos se exponen los requisitos de proyecto obtenidos de las necesidades identificadas en cada etapa del ciclo de vida del robot funcional. La sección tres se presenta la aplicación de despliegue de la función de calidad y la descripción de las matrices asociadas con la metodología. En la sección cuatro se presenta la fase de 
desarrollo y construcción del robot para finalizar con la presentación de resultados y conclusiones del trabajo.

\section{REQUISITOS DEL USUARIO FINAL DEL ROBOT}

En este apartado se definen los requerimientos del usuario, se organizan y se estructuran de cara al planteamiento de la solución.

\subsection{Ciclo de vida del robot e identificación de los usuarios}

Para definir las necesidades de los usuarios, asociados al proyecto, es preciso identificar los clientes de cada una de las etapas del ciclo de vida [7]. En la Figura 1, se muestra las etapas del ciclo de vida consideradas para el proyecto "robot para aplicaciones agrícolas" y los clientes asociados a cada una de ellas. Las necesidades son dadas para cada etapa después de un estudio directo de la voz de los clientes [8].

Definir exactamente las necesidades de los clientes en cada una de las etapas de ciclo de vida es fundamental para aplicar cualquier metodología de diseño del proyecto.

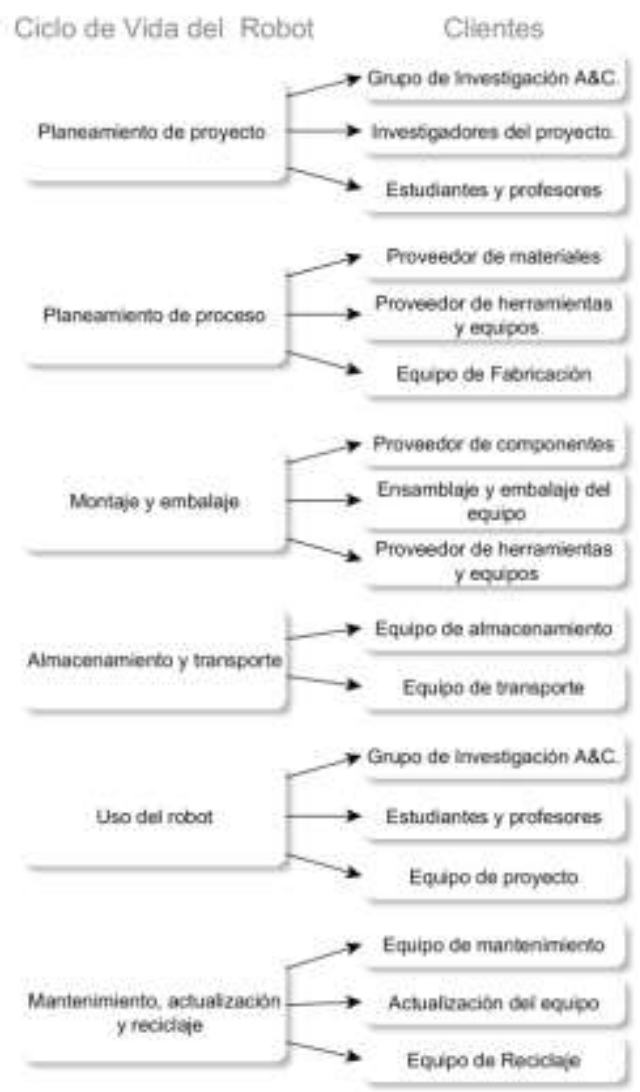

Figura 1. Relación de clientes con el ciclo de vida del robot para aplicaciones agrícolas. Fuente. Elaboración propia.
La definición de las necesidades requiere de un estudio enfocado en cada una de las áreas que abarcan el desarrollo del proyecto. La mejor manera de establecer las necesidades es con una investigación directa de los usuarios finales, a través de técnicas estructuradas de investigación de mercado. Las necesidades identificadas para el desarrollo del robot para aplicaciones agrícolas son presentadas en la Tabla 1.

Tabla 1. Necesidades relacionadas con el robot para aplicaciones agrícolas.

\begin{tabular}{|c|c|}
\hline \multicolumn{2}{|c|}{ Necesidades asociadas al robot de aplicaciones agrícolas } \\
\hline Ciclo de Vida & Necesidades de los Usuarios \\
\hline $\begin{array}{l}\text { Planeamiento de } \\
\text { proyecto }\end{array}$ & $\begin{array}{l}\text { Espacio de trabajo adecuado para } \\
\text { cultivos en interiores. }\end{array}$ \\
\hline $\begin{array}{l}\text { Planeamiento de } \\
\text { proyecto }\end{array}$ & $\begin{array}{l}\text { Garantizar las mismas condiciones de } \\
\text { trabajo para un periodo de tiempo } \\
\text { definido. }\end{array}$ \\
\hline $\begin{array}{l}\text { Planeamiento de } \\
\text { proyecto }\end{array}$ & $\begin{array}{l}\text { Capacidad de proporcionar el mismo } \\
\text { resultado para cada ciclo de operación. }\end{array}$ \\
\hline $\begin{array}{l}\text { Planeamiento de } \\
\text { proyecto }\end{array}$ & $\begin{array}{l}\text { Bajo costo de fabricación y de forma } \\
\text { escalable. }\end{array}$ \\
\hline $\begin{array}{l}\text { Planeamiento de } \\
\text { proyecto }\end{array}$ & $\begin{array}{l}\text { Estructura que facilite la precisión y } \\
\text { proporcione alta estabilidad. }\end{array}$ \\
\hline $\begin{array}{l}\text { Planeamiento de } \\
\text { proyecto }\end{array}$ & $\begin{array}{l}\text { Proporcionar un prototipo virtual para } \\
\text { análisis y simulaciones. }\end{array}$ \\
\hline $\begin{array}{l}\text { Planeamiento de } \\
\text { proceso }\end{array}$ & $\begin{array}{l}\text { Trabajar con las maquinas disponibles } \\
\text { en el laboratorio. }\end{array}$ \\
\hline $\begin{array}{l}\text { Planeamiento de } \\
\text { proceso }\end{array}$ & $\begin{array}{l}\text { Control correctamente sintonizado y } \\
\text { tolerante a interferencias y } \\
\text { perturbaciones. }\end{array}$ \\
\hline $\begin{array}{l}\text { Montaje } \\
\text { embalaje }\end{array}$ & $\begin{array}{l}\text { Usar para el ensamble herramientas } \\
\text { comunes. }\end{array}$ \\
\hline $\begin{array}{l}\text { Almacenamiento } \\
\text { y transporte }\end{array}$ & Fácil de trasportar y embalar. \\
\hline $\begin{array}{l}\text { Almacenamiento } \\
\text { y transporte }\end{array}$ & $\begin{array}{l}\text { Materiales resistentes para soportar } \\
\text { traslados. }\end{array}$ \\
\hline Uso del robot & $\begin{array}{l}\text { Robot visualmente agradable y diseño } \\
\text { relacionado con el entorno de trabajo. }\end{array}$ \\
\hline Uso del robot & Bajo consumo de energía. \\
\hline Uso del robot & $\begin{array}{l}\text { Adaptable para ejecutar diferentes } \\
\text { tareas. }\end{array}$ \\
\hline Uso del robot & $\begin{array}{l}\text { Tecnológicamente compatible para } \\
\text { adherir nuevas tecnologías. }\end{array}$ \\
\hline Uso del robot & Operación con bajo ruido y vibraciones. \\
\hline $\begin{array}{l}\text { Mantenimiento, } \\
\text { actualización } \\
\text { y reciclaje }\end{array}$ & $\begin{array}{l}\text { Componentes estándar } \\
\text { comercialmente asequibles. }\end{array}$ \\
\hline $\begin{array}{l}\text { Mantenimiento, } \\
\text { actualización } \\
\text { y reciclaje }\end{array}$ & Componentes fáciles de intercambiar. \\
\hline $\begin{array}{l}\text { Mantenimiento, } \\
\text { actualización } \\
\text { y reciclaje }\end{array}$ & Facilidad para desmontar. \\
\hline $\begin{array}{l}\text { Mantenimiento, } \\
\text { actualización } \\
\text { y reciclaje }\end{array}$ & Fácil de descartar. \\
\hline
\end{tabular}

Fuente. Elaboración propia. 
Cada fase del ciclo de vida del robot funcional presenta unas necesidades específicas de satisfacer. En la fase de planeamiento del proyecto se especifican las necesidades requeridas os especificaciones deseadas para el robot funcional en la ejecución tareas de agricultura. La aplicación de agricultura contemplada para el trabajo del robot, consiste en asistir de forma autónoma un cultivo de plantas usadas en la "arquitectura sustentable" con la posibilidad de obtener escalamiento de los cultivos. Otros conjuntos de necesidades son obtenidos de la fase de fabricación y ensamble. Estas necesidades definirán desde el diseño, el tipo de herramientas a utilizar, selección de partes estandarizadas y la manera de hacer tareas de mantenimiento. Finaliza con la definición de necesidades relacionadas a la proyección de vida útil donde se crean posibilidades de actualización e inclusión de nuevas tecnologías y como realizar el descarte o reutilización de partes en otros proyectos.

\subsection{Requisitos de proyecto}

Con los procedimientos realizados en la sección anterior para definir las necesidades asociadas al proyecto presentadas en la Tabla 1, y teniendo en cuenta que esa información reúne las necesidades de todas las etapas de ciclo de vida, es necesario hacer un mapeo de ellas para transformarlas en requisitos de proyecto.

El proceso de mapear las necesidades de los clientes a requisitos de proyecto es hecho para definir las propiedades físicas finales del robot. La definición correcta de estas propiedades físicas garantiza que se satisfagan todas las necesidades y especificaciones deseadas. Un lenguaje de ingeniería es usado para definir cada uno de los requisitos de proyecto. Utilizando este lenguaje permite que cada requisito de proyecto sea asociado a una característica técnica o expresión medible y verificable [9].

Los requerimientos de proyecto que representan las necesidades son presentados en la Tabla 2.

\subsection{Diagrama de Mudge}

Con los requisitos definidos en la Tabla 2, la siguiente actividad en la metodología, es establecer el grado de importancia o peso de cada requerimiento. Los requerimientos definidos representan las necesidades, pero algunas de estos demandan un nivel de atención mayor y en ese orden, se debe emplear una técnica correcta para definir el grado de importancia.

El diagrama de Mudge es una herramienta que permite la comparación de los requerimientos de proyecto con el objetivo de generar una jerarquía de ellos, de acuerdo a su grado de importancia y relevancia en el proyecto.
La comparación en el diagrama de Mudge ha sido realizada atribuyendo un valor en cada evaluación de las funciones. El método consiste en responder dos preguntas cuando se hace una comparación entre dos funciones. 1) ¿Cuál de las dos funciones es más importante? 2) Dentro de una escala predefinida ¿cuál es el valor de importancia?. La escala utilizada para definir el grado de importancia contempla valores entre 1 y 5 , donde 5 es el mayor y 1 el menor grado de importancia. Estos valores son representados por las letras A,B,C,D,E con valores respectivos de 5,4,3,2,1.

En la Figura 2 se presenta el diagrama de Mudge. Se puede apreciar en la parte inferior izquierda, la escala predeterminada de eficiencia para definir el grado de importancia. Existe una columna que almacena el resultado de sumar los valores obtenidos de la evaluación de cada requerimiento llamada "suma" y su equivalencia en porcentaje [10].

Tabla 2. Requisitos de proyecto.

\begin{tabular}{|c|l|}
\hline \multicolumn{2}{|c|}{ Requisitos de proyecto } \\
\hline Funciones & \multicolumn{1}{|c|}{ Requerimientos del proyecto } \\
\hline 1 & Fabricación de bajo costo. \\
\hline 2 & Estructura resistente. \\
\hline 3 & Herramienta intercambiable. \\
\hline 4 & Fácil mantenimiento. \\
\hline 5 & Área de trabajo apropiada. \\
\hline 6 & Visualmente agradable. \\
\hline 7 & Consumo de energía bajo. \\
\hline 8 & Alta precisión. \\
\hline 9 & $\begin{array}{l}\text { Nivel de protección adecuado para el } \\
\text { entorno de trabajo. }\end{array}$ \\
\hline 10 & $\begin{array}{l}\text { Control de posición y seguimiento de } \\
\text { trayectoria. }\end{array}$ \\
\hline 11 & Fácil fabricación. \\
\hline
\end{tabular}

Fuente. Elaboración propia.

\subsection{Diagrama de Pareto}

Para un mejor análisis de los resultados obtenidos después de aplicar el diagrama de Mudge, se presenta en la Figura 4 el diagrama de Pareto. Este diagrama es una herramienta para análisis gráfico que facilita la selección de los requerimientos más relevantes del problema, para enfocar los recursos en la satisfacción de ellos. Con el análisis podemos llegar a la conclusión que el $20 \%$ de las causas son responsables del $80 \%$ de los resultados y que existen una cantidad de actividades triviales que debemos identificar para focalizar los recursos en las actividades que más influencia tienen. 


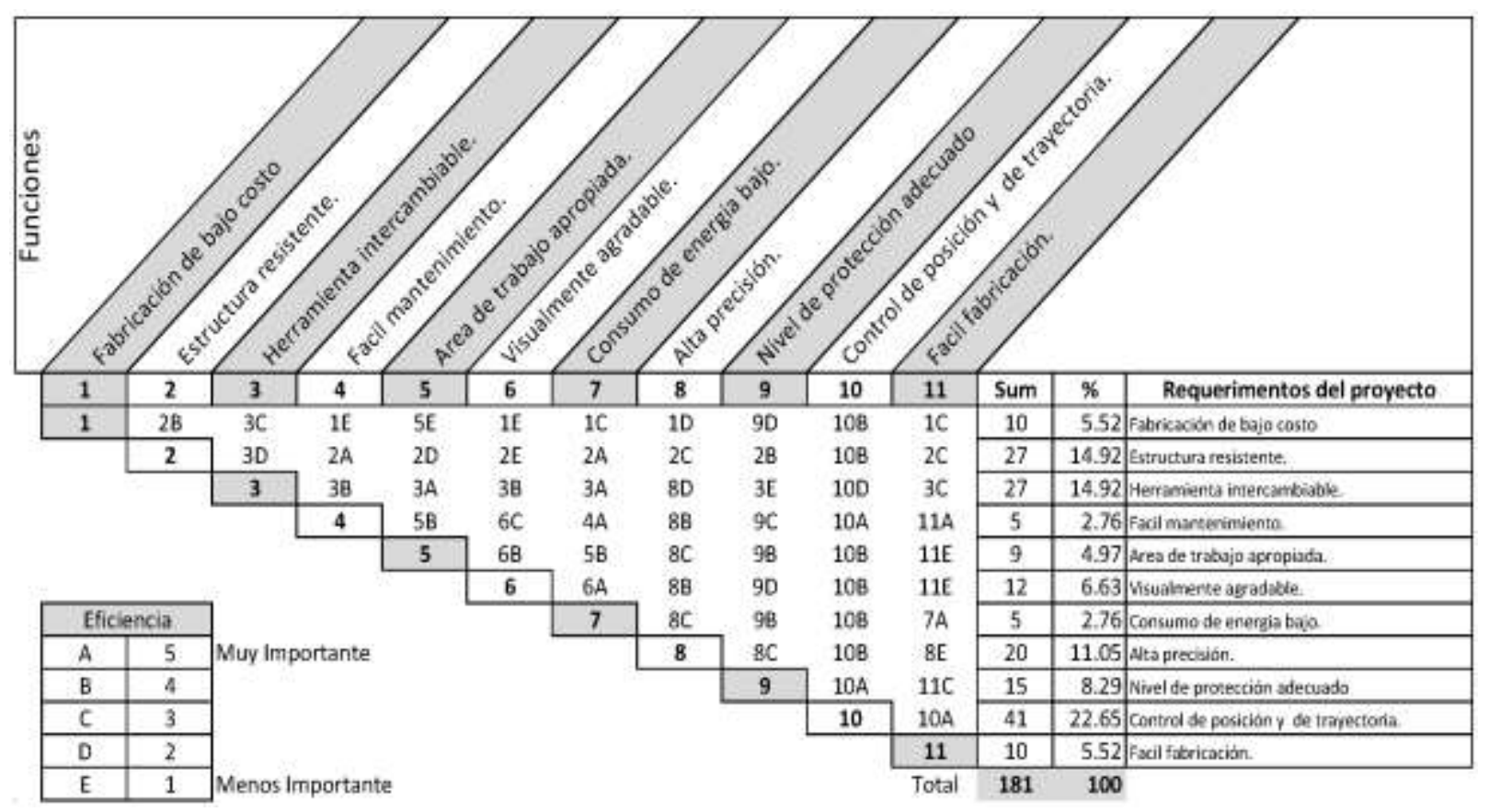

Figura 2. Diagrama de Mudge para el robot para aplicaciones agrícolas. Fuente. Elaboración propia.

El diagrama de Pareto es construido con la información contenida en la Figura 3, que resume los cálculos de análisis necesarios para construir el diagrama. La columna \% es obtenida por medio de la ecuación (1) usando los valores del diagrama de Mudge. La columna "acumulado" se obtiene a través de la ecuación (2).

$$
\%=\frac{\text { Suma }}{\text { Total }} \cdot 100
$$

$$
\text { Acumulado }=\%+\text { Acumulado }_{\text {Anterior }}
$$

\begin{tabular}{|c|c|c|c|c|}
\hline Funciones & Requerimientos del proyecto & $\%$ & Acuinulado & Suma \\
\hline 10 & Control de posición y de trayectoria. & 22.65 & 22.65 & 41 \\
\hline 2 & Estructura resistente. & 14.92 & 37.57 & 27 \\
\hline 3 & Herramienta intercambiable. & 14.92 & 52.49 & 27 \\
\hline 8 & Alts preúsión & 11.05 & 63.54 & 20 \\
\hline 9 & Nivel de grotección adecuada & 8.29 & 71.83 & 25 \\
\hline 6 & Visualmente agradable. & 6.63 & 78.46 & 12 \\
\hline 1 & Fabricación de baja costa. & 5.52 & 83.98 & 10 \\
\hline 11 & Fácil fabritación. & 5.52 & 89.5 & 10 \\
\hline 5 & Area de trabajo apropiada. & 4.57 & 94.47 & 9 \\
\hline 4 & Fàcil manterimiento. & 2.77 & 97.24 & 5 \\
\hline 7 & Consumb do energia bajo. & 2.76 & $I D O$ & 5 \\
\hline
\end{tabular}

Figura 3. Resumen de los cálculos en el análisis de Pareto.

El objetivo de utilizar el diagrama de Pareto es proporcionar una forma alterna para observar el grado de importancia de cada requerimiento. Este permite enfocar recursos y esfuerzos en la satisfacción de requerimientos que acercan a la solución deseada. También ayuda en la toma de decisiones relacionadas con el desarrollo del proyecto.

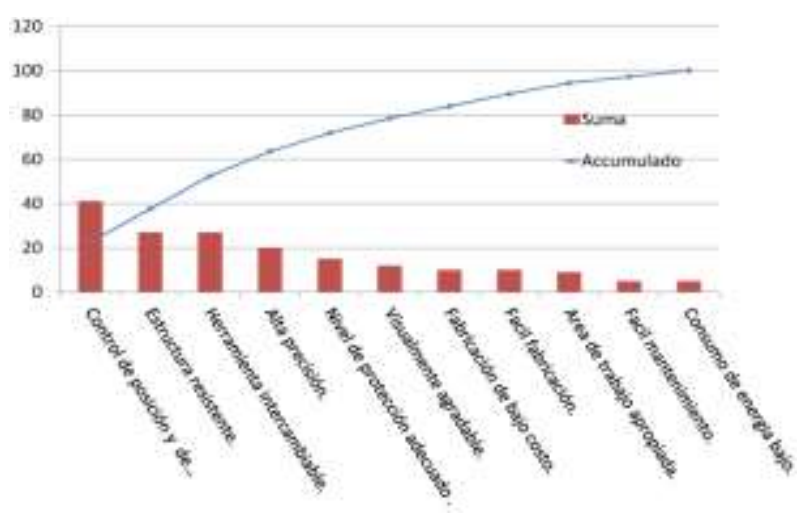

Figura 4. Diagrama de Pareto del robot para aplicaciones agrícolas.

Las barras en la Figura 4 representan cada uno de los requerimientos del robot y el valor resultante de la comparación con los otros requerimientos obtenidos con en el diagrama de Mudge. La curva indica los acumulados que van ascendiendo hasta el valor de 100 por ciento.

La ventaja de aplicar este método radica en que con una observación sobre la Figura 4 se puede deducir que, si se aplica el mayor de los esfuerzos y recursos en la satisfacción de los primeros 6 requerimientos, el proceso está un 80 por ciento más cerca de encontrar la solución a las necesidades planteadas por los clientes del robot agrícola. 


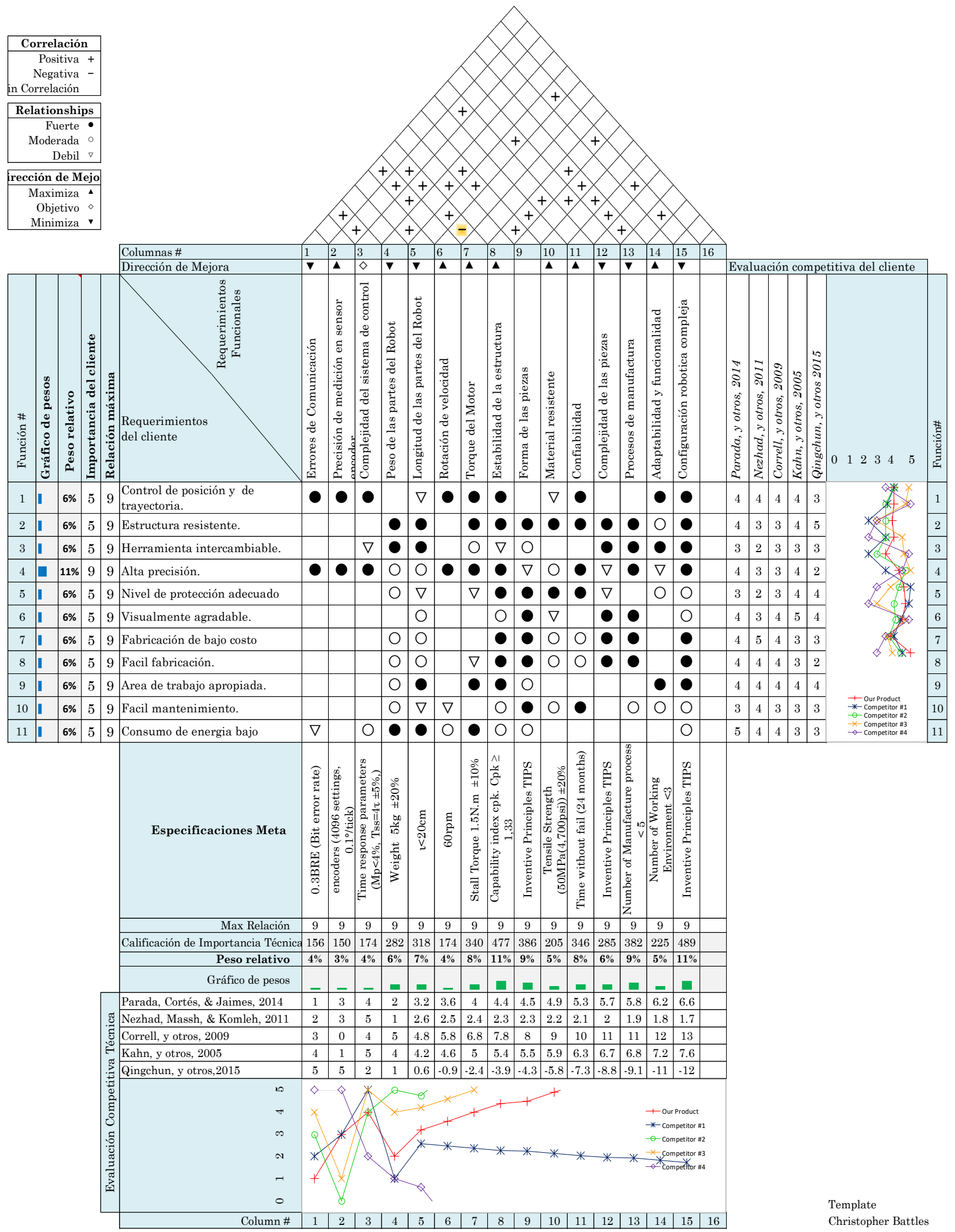

Figura 5. Estructura matriz de la casa de la calidad de QFD. Fuente. Elaboración propia. 


\section{APLICACIÓN DESPLIEGUE DE LA FUNCIÓN DE CALIDAD}

Para continuar con la fase de estudio y diseño del robot agrícola, se emplea la metodología de despliegue de la función de calidad QFD, como método para guiar las siguientes actividades en el desarrollo del robot agrícola. Esta metodología recopila los requerimientos y necesidades del robot y las despliega en un conjunto de características técnicas y operativas conducentes a crear un concepto del producto que satisface los requerimientos [11].

La metodología más que proporcionar datos cuantitativos, proporciona información de carácter cualitativo, fundamental para generar el concepto de diseño y explorar múltiples soluciones, que se adapten a las características técnicas con las que se cuenta para la ejecución de este proyecto. Estos elementos cualitativos permiten satisfacer en gran medida los requerimientos conservando el control de recursos tales como costos y tiempo para ejecución del proyecto.

Otro aspecto importante que motiva la aplicación de la metodología es que permite hacer un estudio comparativo del proyecto con desarrollos similares, fortaleciendo el concepto de diseño por incluir la evaluación de la competencia en la etapa de estudio [12].
En la Figura 5 se presenta el esquema general QFD y las matrices asociadas a la casa de la calidad. El surgimiento de este método fue atribuido a la necesidad de incorporar un sistema de calidad en los diseños y definir los tiempos para realizar el control de procesos. Con la elaboración de la casa de la calidad, argumentos como las necesidades de los clientes, análisis de proyectos similares, necesidades del equipo de fabricación y correlación entre los requerimientos de proyecto son incorporados y trasladados a la solución final [8]. A continuación, se presentan cada una de las matrices que componen el despliegue de la función de calidad.

\subsection{Matriz de requerimientos del proyecto y análisis de competencia}

El origen y estudio de los requerimientos del proyecto para el robot agrícola fueron presentados en el apartado anterior. Estos requerimientos conforman la primera matriz de la metodología. La matriz de importancia, es conformada por los pesos de los requerimientos del proyecto definidos en una escala de 1 a 5 , también obtenidos en el apartado anterior con la aplicación del diagrama de Mudge.

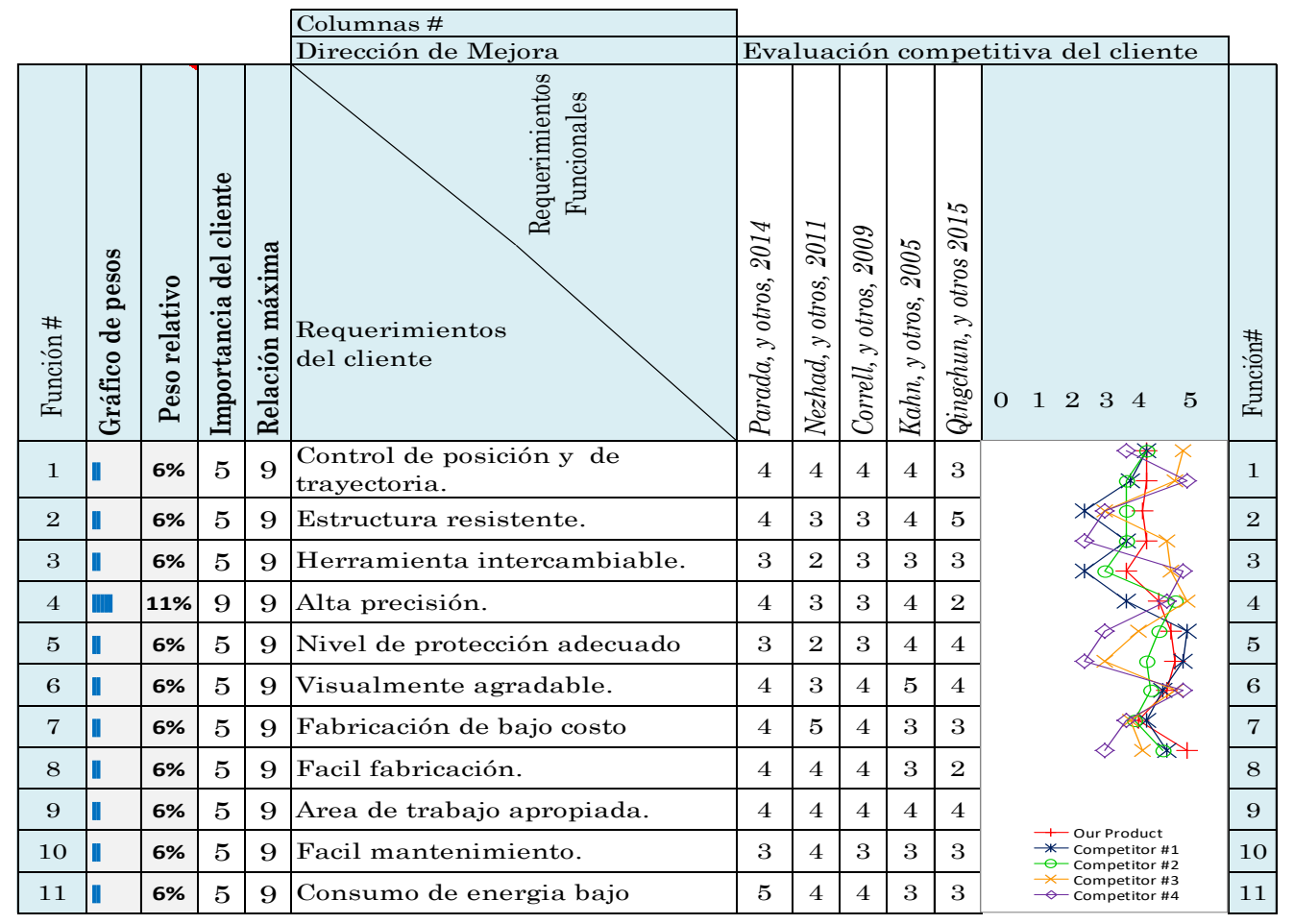

Figura 6. Requerimientos del proyecto y Benchmarking competitivo. Fuente. Elaboración propia. 
En la Figura 6 se presenta la matriz de requerimientos, el grado de importancia y el análisis de la competencia. Se hace un estudio comparativo de seis proyectos que mantienen relación con el robot a desarrollar. En ese estudio fue incluido un primer prototipo de brazo robótico para evaluar el comportamiento frente a cada uno de los requerimientos [13]. El resultado de este benchmarking competitivo se resume en evaluaciones dentro de una escala de 1 (menor evaluación) a 5 (mejor evaluación), y presentado en la Figura 6.

\subsection{Requisitos de producto}

Los requerimientos de producto para el robot sembrador, son definidos en función de criterios de ingeniería, que permiten verificar el cumplimiento de cada una de las necesidades a través de mediciones sobre estas variables. La definición de estos requerimientos en unidades de ingeniería permite crear expresiones medibles para cuantificación y verificación. En cada requisito de proyecto puede definirse uno o más elementos de producto (sistema, sub-sistema, componente, característica o función) en el que cada elemento tiene asignada una variable medible también conocida como característica técnica de calidad del producto $[14,8]$.

Para determinar los requerimientos del proyecto, de forma adecuada, se busca aprovechar la relación que existe entre TRIZ (Tieoriya Riesheniya Izobrietatielskij Zadach) y la metodología QFD (Quality Function Developed), principalmente para apoyar la definición de las especificaciones del proyecto y la eliminación de las contradicciones asociadas a los parámetros de ingeniería. La Teoría para Resolver Problemas de Inventiva TRIZ o su acrónimo en inglés TIPS (theory of inventive problem solving), proporciona una lista de parámetros de ingeniería comunes en muchos problemas de diseño que conducen a crear una característica medible de calidad. También la teoría proporciona unos principios de solución inventivos útiles para encontrar una solución análoga en problemas con algún grado de acoplamiento. En la Figura 7 se presenta los requisitos de proyecto.

\subsection{Matriz de correlación}

La matriz de correlación tiene una función importante dentro de la metodología, a través de ella se identifican las especificaciones de desempeño del sistema que tienen algún tipo de acoplamiento. La identificación temprana de los requisitos acoplados, permite explorar soluciones que conducen a eliminar la correlación. La evaluación de la matriz está hecha con base en una interrelación de los requisitos de producto para identificar el grado de dependencia. Se identifica no solo la relación sino también la dirección de esa relación. Se emplean tres criterios para realizar esta correlación:
- Correlación positiva: Es la relación de dos requisitos de producto que ambos aumentan o disminuyen simultáneamente.

- Correlación negativa: Es la relación de dos requisitos de producto que presentan una dependencia directa. Es una relación que es inversamente proporcional y constante que se interpreta que al aumentar ese requerimiento de proyecto afecta disminuyendo el otro.

- Correlación nula: Cuando no existe ninguna relación clara de dependencia de esos dos requisitos de producto.

En la Figura 8 se presentan los diferentes criterios utilizados para evaluar y generar las matrices de relacionamiento, dirección y correlación de la casa de calidad o método QFD.

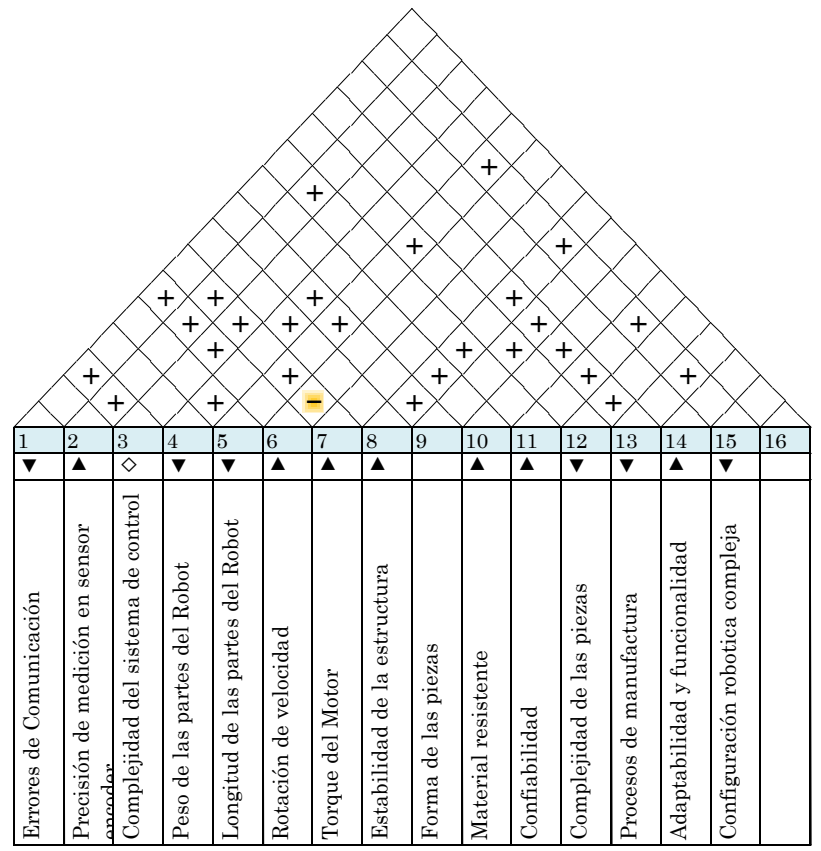

Figura 7. Requisitos de producto y matriz de correlación para el robot de aplicaciones agrícolas. Fuente. Elaboración propia.

\begin{tabular}{|l|}
\hline Correlación \\
\hline Positiva + \\
Negativa ? \\
Sin Correlación \\
\hline \multicolumn{2}{|c|}{ Relationships } \\
\hline \multicolumn{2}{|c|}{ Fuerte ? } \\
Moderada ? \\
Debil ? \\
\hline Direción de Mejora \\
\hline Maximiza ? \\
Objetivo ? \\
Minimiza ? \\
\hline
\end{tabular}

Figura 8 Criterio de evaluación para las matrices de correlación, relacionamiento y dirección. Fuente. Elaboración propia. 
La correlación es presentada en la Figura 7 y conforma el techo de la casa de la calidad. Se puede observar una correlación negativa entre dos requerimientos, el sistema precisa de motores de alto torque, pero a su vez dar velocidad para la rotación de las articulaciones.

\subsection{Matriz de relacionamiento}

La matriz de relacionamiento indica de forma cualitativa, en cuánto cada requisito de proyecto afecta cada requerimiento de cliente. Esta cualificación es realizada a través de un grado de relacionamiento dividido en tres criterios:

- Fuertemente relacionado.

- Relacionado moderadamente.

- Débilmente relacionado.

En la Figura 9 se presenta la matriz de relacionamiento generada para los requisitos del cliente y los requisitos de producto.

\subsection{Especificaciones meta}

Las especificaciones meta para el proyecto son presentadas en la Tabla 3.

Las especificaciones se interpretan como acotaciones específicas del proyecto y representan las características de calidad perseguidas con el robot. Para fijar estas especificaciones se contemplan los recursos técnicos y físicos que se disponen para el desarrollo del proyecto.
Tabla 3. Especificaciones Meta.

\begin{tabular}{|l|l|}
\hline \multicolumn{2}{|c|}{ Especificaciones (Meta) } \\
\hline Requerimientos & Especificaciones \\
\hline Errores de Comunicación & $0.3 \mathrm{BRE}$ (Tasa error en Bit) \\
\hline $\begin{array}{l}\text { Precisión de medición en } \\
\text { sensor encoder }\end{array}$ & $\begin{array}{l}12 \text {-bit Encoder magnéticos } \\
(4096 \quad \text { Configuración, } \\
0.1^{\circ} / \text { tick) }\end{array}$ \\
\hline $\begin{array}{l}\text { Complejidad del sistema de } \\
\text { control }\end{array}$ & $\begin{array}{l}\text { Parámetros de tiempo de } \\
\text { respuesta } \\
\left(\mathrm{Mp}<4 \%, \text { Tss }=4 \_\right)\end{array}$ \\
\hline Peso de las partes del Robot & Peso 5kg $\pm 20 \%$ \\
\hline $\begin{array}{l}\text { Longitud de las partes del } \\
\text { Robot }\end{array}$ & Longitud $<20 \mathrm{~cm} \pm 10 \%$ \\
\hline Rotación de velocidad & 60rpm $\pm 10 \%$ \\
\hline Motor torque. & Torque 1.5N.m \\
\hline Estabilidad de la estructura & - \\
\hline Forma de las piezas & $\begin{array}{l}\text { Principios inventivos } \\
\text { basados en TIPS. }\end{array}$ \\
\hline Material resistente & $\begin{array}{l}\text { Resistencia a la tracción } \\
(50 M P a(4,700 p s i))\end{array}$ \\
\hline Confiabilidad & Tiempo sin fallas (24 meses) \\
\hline Complejidad de las piezas & $\begin{array}{l}\text { Principios inventivos } \\
\text { basados en TIPS. }\end{array}$ \\
\hline Procesos de manufactura & $\begin{array}{l}\text { Número de procesos de } \\
\text { manufactura }<5\end{array}$ \\
\hline $\begin{array}{l}\text { Adaptabilidad } \\
\text { funcionalidad }\end{array}$ & $\begin{array}{l}\text { Número de entornos de } \\
\text { trabajo <3 }\end{array}$ \\
\hline $\begin{array}{l}\text { Configuración } \\
\text { compleja }\end{array}$ & $\begin{array}{l}\text { Principios inventivos } \\
\text { basados en TIPS. }\end{array}$ \\
\hline
\end{tabular}

Fuente. Elaboración propia.

\begin{tabular}{|c|c|c|c|c|c|c|c|c|c|c|c|c|c|c|c|c|}
\hline Columnas \# & 1 & 2 & 3 & 4 & 5 & 6 & 7 & 8 & 9 & 10 & 11 & 12 & 13 & 14 & 15 & 16 \\
\hline Dirección de Mejora & $\nabla$ & $\mathbf{A}$ & $\diamond$ & $\nabla$ & $\mathbf{V}$ & $\mathbf{\Delta}$ & $\mathbf{A}$ & $\Delta$ & & $\Delta$ & $\Delta$ & $\nabla$ & $\mathbf{V}$ & $\mathbf{\Delta}$ & $\nabla$ & \\
\hline $\begin{array}{l}\text { Requerimientos } \\
\text { del cliente }\end{array}$ & 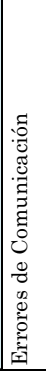 & 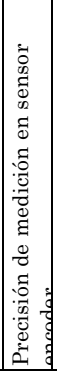 & 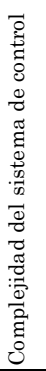 & 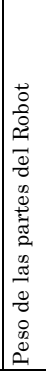 & 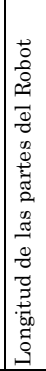 & 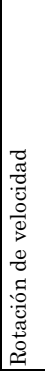 & 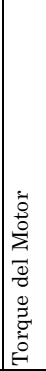 & 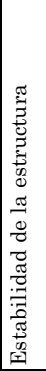 & 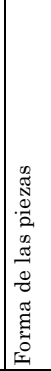 & 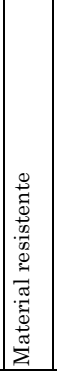 & 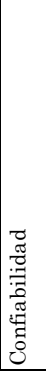 & 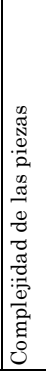 & 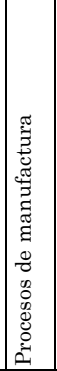 & 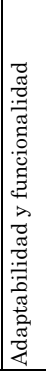 & 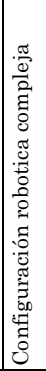 & \\
\hline $\begin{array}{l}\text { Control de posición y de } \\
\text { trayectoria. }\end{array}$ & • & ○ & $\bullet$ & & $\nabla$ & ○ & $\bullet$ & - & & $\nabla$ & - & & & - & - & \\
\hline Estructura resistente. & & & & 0 & 0 & & 0 & $\bullet$ & - & 0 & 0 & 0 & 0 & 0 & 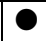 & \\
\hline Herramienta intercambiable. & & & $\nabla$ & 0 & 0 & & 0 & $\nabla$ & O & & & - & 0 & - & - & \\
\hline Alta precisión. & 0 & 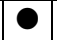 & 0 & O & 0 & 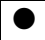 & 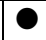 & 0 & $\nabla$ & $O$ & 0 & $\nabla$ & - & $\nabla$ & 0 & \\
\hline Nivel de protección adecuado & & & & 0 & $\nabla$ & & $\nabla$ & 0 & $\bullet$ & 0 & 0 & $\nabla$ & & 0 & 0 & \\
\hline Visualmente agradable. & & & & & $\mathrm{O}$ & & & $\mathrm{O}$ & • & $\nabla$ & & 0 & 0 & & 0 & \\
\hline Fabricación de bajo costo & & & & 0 & O & & & 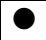 & $\bullet$ & O & $\mathrm{O}$ & 0 & 0 & & 0 & \\
\hline Facil fabricación. & & & & O & O & & $\nabla$ & 0 & - & O & O & 0 & - & & 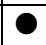 & \\
\hline Area de trabajo apropiada. & & & & 0 & 0 & & 0 & $\bullet$ & $\mathrm{O}$ & & & & & 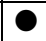 & 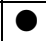 & \\
\hline Facil mantenimiento. & & & & 0 & $\nabla$ & $\nabla$ & & O & $\bullet$ & O & 0 & & O & O & O & \\
\hline Consumo de energia bajo & $\nabla$ & & O & 0 & 0 & $\mathrm{O}$ & 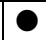 & O & $\mathrm{O}$ & & & & & & 0 & \\
\hline
\end{tabular}

Figura 9. Matriz de relacionamiento para el robot de aplicaciones agrícolas. Fuente. Elaboración propia. 


\subsection{Cuantificación de los requisitos del producto}

En esta matriz se presenta el resultado obtenido de aplicar la técnica QFD para encontrar el mejor camino que conduzca a satisfacer las necesidades del cliente. En la Figura 10 se presenta la matriz.
$\mathrm{Al}$ analizar la figura se puede observar el peso y grado de importancia de los requerimientos del producto. Esta información es relevante para el planeamiento y desarrollo del proyecto. Con la información extraída de la metodología QFD se realizó una asignación equitativa de los recursos y se enfocaron los esfuerzos en los requerimientos que presentan mayor relevancia [15].

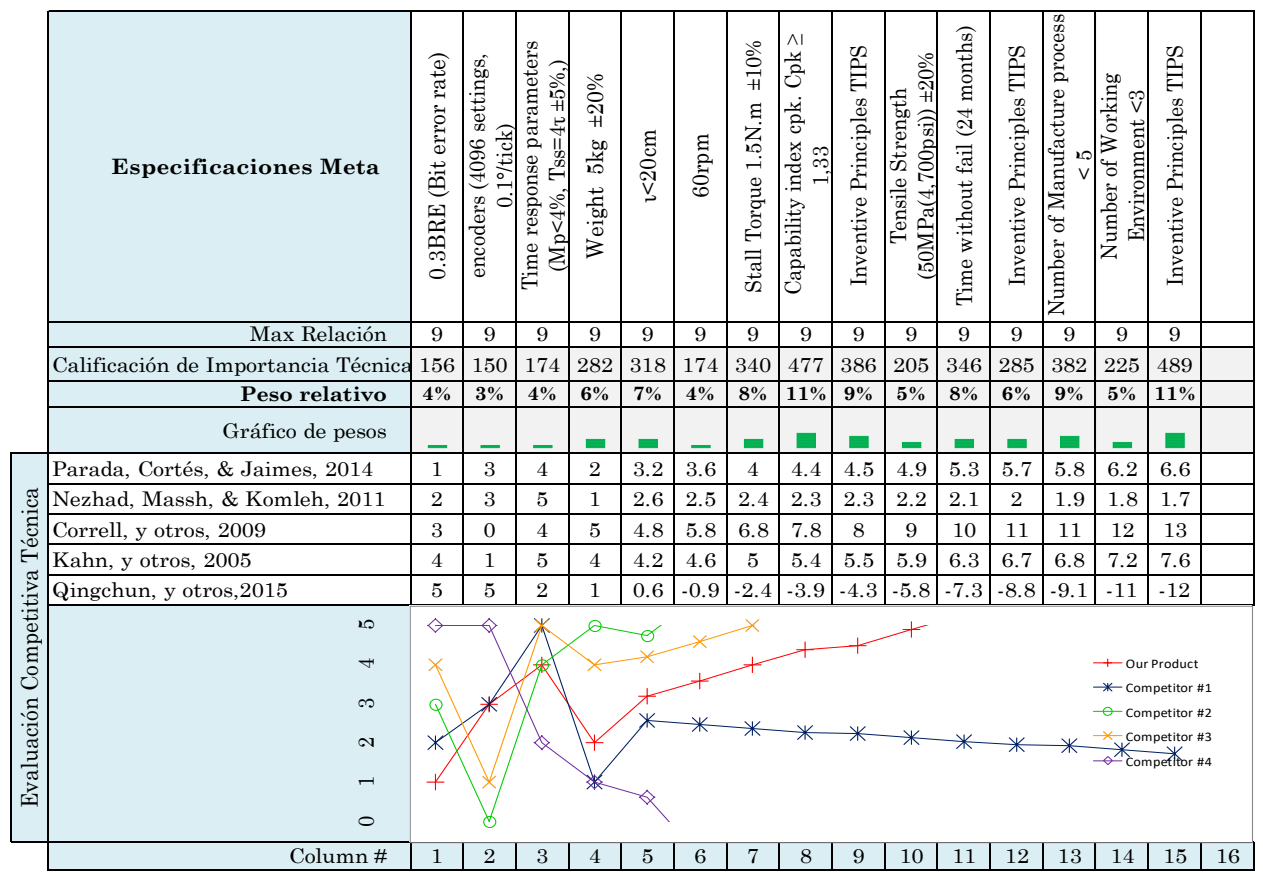

Figura 10. Matriz de cuantificación de los requisitos del producto. Fuente. Elaboración propia.

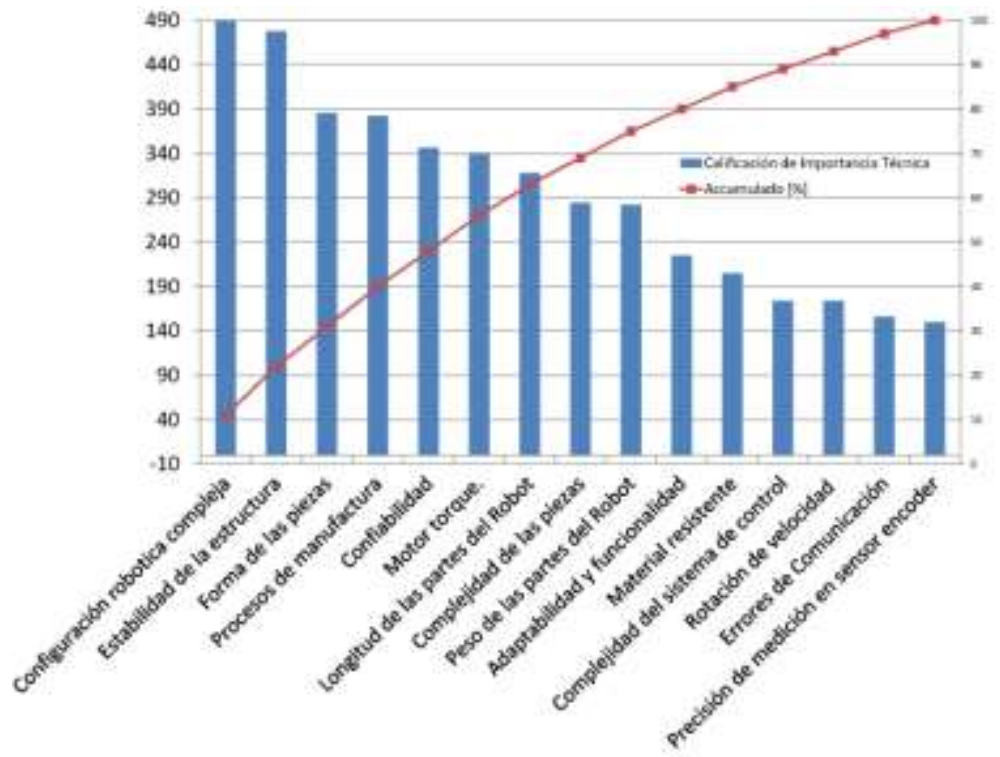

Figura 11. Resultado en orden de importancia de los requerimientos funcionales con metodología QFD. Fuente. Elaboración propia. 
Las características de producto se presentan en orden de importancia a través de diagrama de Pareto presentado en la Figura 11, para facilitar el análisis. En el diagrama se observa que, si se enfocan los recursos en las causas vitales, características con mayor grado de relevancia, esto permitirá lograr la mayoría de los beneficios potenciales.

\section{DESARROLLO DEL PROYECTO}

En el desarrollo del proyecto se toman los resultados de la matriz QFD y se abordan con profundidad cada uno de los requerimientos funcionales, con el objeto de generar el diseño conceptual que contenga la ingeniería de detalle asociada a cada solución [16]. Para presentar la ingeniería de detalle de la solución, se construye un prototipo virtual con software de modelado CAD. Se definen aspectos de apariencia, dimensiones y propiedades físicas para obtener el prototipo con las características físicas reales que el robot adquirirá en su fabricación. El prototipo virtual final obtenido es mostrado en la Figura 12.
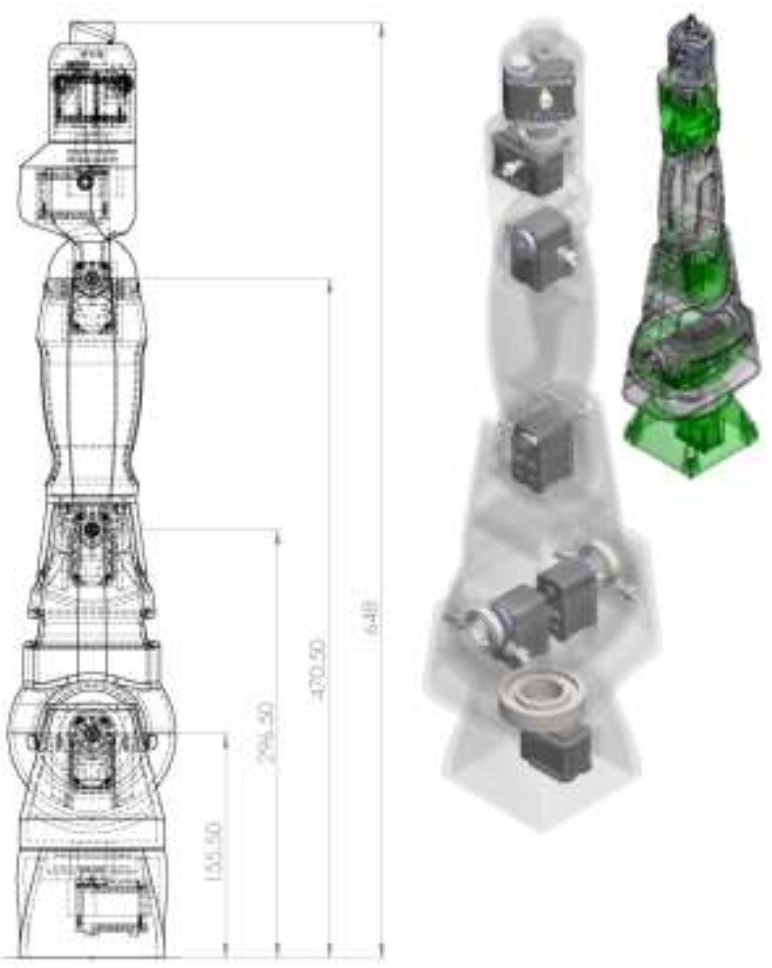

Figura 12. Prototipo virtual del brazo robótico. Fuente. Elaboración propia.

La herramienta CAD permite modelar piezas y conjuntos para extraer planos, lista de materiales y cualquier tipo de información necesaria para la construcción y desarrollo del prototipo. El proceso de crear el modelo CAD consiste en formalizar la idea dibujando las piezas y creando ensambles para obtener simulaciones, planos, desarrollos y distribución.

En la fabricación del prototipo del brazo robótico se empleó el proceso de manufactura FDM (Fused Deposition Modeling). El equipo empleado para este proceso de fabricación es la impresora 3D MakerBot Replicator Z18.

En la Figura 13 se presenta el espacio trabajo que abarca el brazo robótico. El volumen del espacio de trabajo es aproximadamente 413892099.81 milímetros cúbicos con una superficie que cubre un área de superficie de 3373138.89 milímetros cuadrados. La carga nominal admisible del robot es de 400 gramos, las velocidades máximas de las articulaciones de los ejes 1, 4, 5 son: 177 grados por segundo, el de las articulaciones de los ejes 2 y 3 son: 207 grados por segundo. La velocidad nominal u objetivo de todas las articulaciones es de 67.5 grados por segundo.

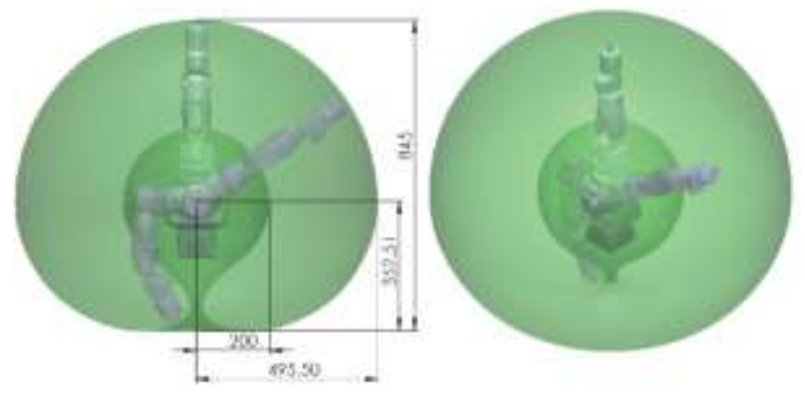

Figura 13. Representación del espacio de trabajo del brazo robótico. Fuente. Elaboración propia.

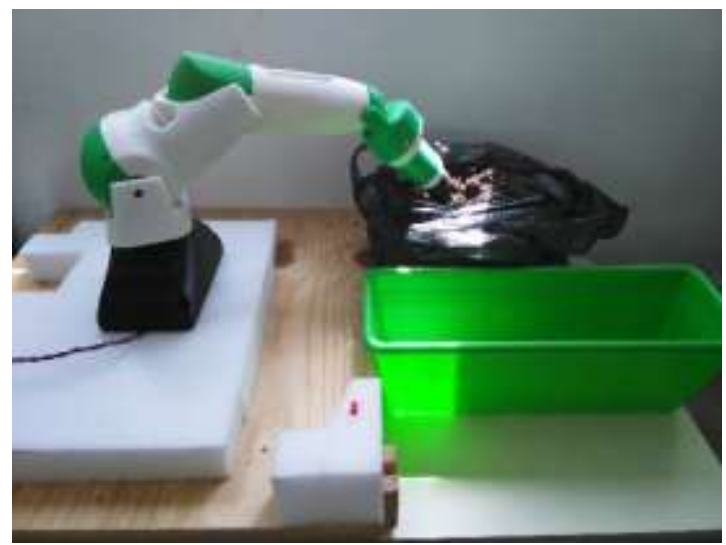

Figura14. Robot recogiendo mezcla de sustrato. Fuente. Elaboración propia.

En la Figura 14 y en la Figura 15 se presenta el robot desarrollado completamente operacional en la etapa de programación. El robot es sometido a pruebas para evaluar el cumplimiento de los requisitos del cliente. 


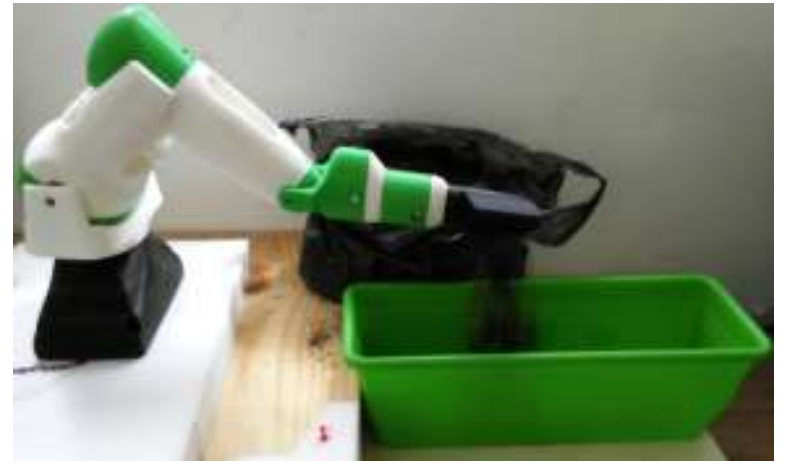

Figura 15. Robot vertiendo mezcla en las materas. Fuente. Elaboración propia.

\section{CONCLUSIONES}

Se implementó una metodología para el desarrollo de un proyecto mecatrónico que incorpora técnicas y metodologías de diseño de producto con el objeto de interpretar las necesidades del proyecto e incorporar soluciones conducentes a satisfacerlas.

La metodología permitida reducir el tiempo de fabricación y realizar mejor uso de los recursos disponibles. Aunque la etapa de diseño consumió un tiempo mayor que el usado en anteriores proyectos, los resultados fueron favorables. Se redujo errores, se exploraron nuevos principios de solución y se consiguió un diseño conceptual escalable tecnológicamente y acoplable a diferentes tareas.

Las técnicas de resolución de problemas aportaron salidas a problemas con grados de acoplamiento que satisfacen los dos requerimientos sin tener pérdidas de desempeño. El producto obtenido, un robot antropomórfico que cumple los requerimientos fijados e incorpora un sistema para el intercambio de herramientas, brindándole adaptabilidad a futuras tareas.

Se evidenció que, a pesar de que la etapa de estudio y diseño demande una parte importante del tiempo total empleado, el balance final es positivo, ya que la metodología retorna beneficios asociados a la reducción de tiempo en la fabricación y ensamble, producto de la focalización de recursos, del aprovechamiento de herramientas y condiciones técnicas y a la reducción de pérdidas en compras innecesarias.

El artículo presenta un abordaje general para el desarrollo de robots, basado en técnicas de resolución de problemas como es la metodología QFD. La integración de técnicas como el diagrama de Mudge, la matriz QFD y la teoría inventiva TRIZ para resolver problemas, garantizan un desarrollo óptimo del proyecto.

\section{REFERENCIAS}

[1] M. F. Maradei, F. Maldonado, C. Gómez, "Aplicación de la ergonomía en el desarrollo de un periférico de entrada y control de datos para discapacitados," Rev. UIS Ing., vol. 8, no. 1, pp. 65-75, 2009.

[2] M, Suell, J. F. Archila, O. Lengerke, "Diseño mecatrónico de un robot tipo agv automated guided vehicle," Rev. UIS Ing., vol. 7, n. 1, pp. 65-76, 2010.

[3] H. González, C. A. Mejía, "Estudio comparativo de tres técnicas de navegación para robots móviles," Rev. UIS Ing., vol. 6, n. 1, pp. 77-84, 2011.

[4] C. A. P. Cortés, C. I. R. Jaimes y J. A. R. Valencia, "Desarrollo de un sistema robótico aplicado a superficies verdes en edificaciones", Revista de Tecnología, vol. 15, pp. 33-42, May. 2016.

[5] L.-K. Chan and M.-L. Wu, "Quality function deployment: A literature review," European journal of operational research, vol. 143, pp. 463-497, Dec. 2002.

[6] K. Masui, T. Sakao, M. Kobayashi and A. Inaba, "Applying quality function deployment to environmentally conscious design", International Journal of Quality \& Reliability Management, vol. 20, pp. 90-106, 2003.

[7] E. Cortés, E. Rodríguez, C. Riaño, R. Bonnard y C. Peña, "Enfoque sobre o desenvolvimento de um robô com arquitetura paralela $5 \mathrm{r}$ para manufatura aditiva", IX Congresso Brasileiro de Engenharia de Fabricação COBEF 2017. Brasil, Jun. 2017.

[8] H. Rozenfeld e others, "Gestão de desenvolvimento de produtos: uma abordagem para a melhoria de processos", Sao Paulo: Saraiva, 2006.

[9] G. \&. B. Ç. Büyüközkan, "Designing a sustainable supply chain using an integrated analytic network process and goal programming approach in quality function deployment," Expert Systems with Applications, vol. 38, pp. 13731-13748, 2011.

[10] H. Kume, E. Vasco y H. Kume, "Herramientas estadísticas básicas para el mejoramiento de la calidad", Editorial Norma, 1992.

[11] H. Rozenfeld, F. A. Forcellini e D. C. Amaral, "Gestão de desenvolvimento de produtos: uma 
referência para a melhoria do processo", Editora Saraiva, 2000.

[12] Y. Akao, "Quality function deployment, Integrating Customer Requirements Into Product Design" 2004.

[13] P. A. C. Parada, C. A. P. Cortés y C. I. R. Jaimes, "Módulo robótico para la clasificación de lulos Solanum Quitoense, implementando visión artificial", INGE CUC, vol. 10, pp. 51-62, 2014.

[14] Y. Akao, Hoshin Kanri: "Policy deployment for successful TQM", SteinerBooks, 2004.

[15] A. \&. D. S. F. C. Nieves Hurtado, "Probabilidad y Estadística para Ingeniería, un enfoque moderno", México DF: McGra-Hill, 2010.

[16] L. A. B. Mascarenhas, J. Oliveira Gomes, A. T. Portela, C. V. Ferreira e L. L. N. Guarieiro, "Utilização da matriz morfológica para desenvolvimento da bancada de testes de válvulas e sedes de válvulas”, 2015. 